\title{
Fabrication and Characterization of Poly Lactic Acid Scaffolds by Fused Deposition Modeling for Bone Tissue Engineering
}

\author{
Mohammad Khodaei ${ }^{1}$, Kamran Amini' ${ }^{2}$, Alireza Valanezhad ${ }^{3 *}$ \\ ${ }^{1}$ Department of Materials Science and Engineering, Golpayegan University of Technology, Golpayegan, Iran \\ (Email: khodaei@gut.ac.ir). \\ ${ }^{2}$ Department of Materials Engineering, Tiran Branch, Islamic Azad University, Isfahan, Iran (Email: \\ kamran_amini1978@,hotmail.com). \\ ${ }^{3}$ Department of Dental and Biomedical Materials Science, Nagasaki University, Nagasaki, Japan. \\ * Corresponding author: E-mail: vala@nagasaki-u.ac.jp,(Alireza Valanezhad)
}

\begin{abstract}
:
Porous biodegradable polymeric scaffolds are a good option for hard tissue repair and bone tissue engineering. In this research, three-dimensional porous poly-lactic acid (PLA) scaffold was fabricated using fused deposition modeling (FDM) method including 30, 50 and $70 \%$ nominal porosity. Study of phases in initial polymeric material and printed scaffolds was done by X-ray diffraction (XRD), and indicate that no significant phase difference has been observed due to the manufacturing process, and the poly-lactic acid retains its crystalline properties. The results of the mechanical properties evaluation by the compression test show that the mechanical properties of the scaffold have decreased significantly with increasing the porosity of scaffold. The microstructure of scaffolds were studied by Scanning Electron Microscope (SEM), and this observation shows that the pores had a regular arrangement and their morphology changes with porosity change. The mechanical properties of the poly-lactic acid scaffolds printed using fused deposition modeling, can be adapted to the surrounding tissue, by porosity change.
\end{abstract}

Keywords: 3D porous scaffold; Fused deposition modeling; Poly-lactic acid; Biodegradable polymer.

\section{Introduction:}

Porous scaffolds are used extensively in tissue engineering to adjust the implant elastic modulus, help to better nutrition the tissue around the implant and remove wastes [1]. The 
pores of the scaffolds help to grow and differentiate the cells and accelerate the healing of damaged tissue. Scaffolds can be made up from ceramic, metal or polymer in terms of application, and can be fabricated with several method such as space holder, replica, gel casting, thermal induction and ... [2]. Lack of controlling the size and morphology of porosities is one of the defects of the mentioned methods. Therefore, rapid prototyping techniques are expanding to improve the microstructure of scaffolds and improve the patient's treatment process [3]. In these methods, the computer model of the three-dimensional porous scaffold is first plotted and, after layer-to-layer separation, each layer is sent to the machine for building or printing. Though so far more than 30 different prototyping techniques have been developed, but they have similar work bases. Fused Deposition Modeling (FDM) is a simple, efficient, and fast method for building porous scaffolds by printing thermoplastic polymers [3, 4].

In this method, the thermoplastic polymer filament (such as polyvinyl alcohol, poly lactic acid, etc.) is heated and melted in the printer's head and, the head of the printer, taking the command from the controller, deposits the polymer melt in the desired position $[5,6]$.

Medical polymers are categorized into biodegradable and non-biodegradable classes. Biodegradable polymers are decomposed after implanting in the body after a while. The decomposition products can be absorbed and used or disposed by human body metabolism. One of the most important biodegradable polymers in medical engineering is Ploy Lactic Acid (a type of poly-alpha-hydroxy ester) that has been used extensively in the medical environment with a degradation time of about 4 months in the body [3]. This biodegradable thermoplastic polymer can be easily processed by fusion techniques, such as the fused deposition modeling [3]. Ploy lactic acid polymer with flexural strength of $97 \mathrm{MPa}$, density of $1.25 \mathrm{~g} / \mathrm{cm}^{3}$, and melting point of $180{ }^{\circ} \mathrm{C}$ is considered a good option for bone implantation [4].

Lin et al. [4] used post-print heating techniques to improve the bond strength of bi-material polymer piece (poly-caproclactone-poly lactic acid) produced by fused deposition modeling and they could improve the tensile strength of pieces by $28 \%$.

Corkion et al. [3] used hydroxyl apatite microspheres to improve the bioactivity of scaffolds made of poly lactic acid by fused deposition modeling, although the presence of hydroxyl apatite microspheres in porous poly lactic acid scaffold did not change the glass temperature difference of the polymer, but its mechanical strength has been reduced and hydroxyl apatite has not been acted as an reinforcement of the mechanical properties of poly lactic acid polymer. 
Grass et al. [7] studied the effect of the parameters of layer thickness, fill density, nozzle diameter and printing rate on the fatigue behavior of poly lactic acid polymer produced by fused deposition modeling. Their research results indicated that the fill density of sample is the most effective parameter on the fatigue behavior of the poly lactic acid produced by the fused deposition modeling.

In this study, the scaffold of biodegradable polymer of poly lactic acid was constructed using fused deposition modeling with ordered pores and, the effect of porosity on the microstructure and mechanical properties of the scaffold was evaluated.

\section{Materials and methods}

\subsection{Fabrication of poly lactic acid porous scaffold by fused deposition modeling}

After drawing the 3D model by SolidWorks 2014 software, the file was saved in stl format [5] and sent to a 3D printer device. The ploy lactic acid polymer filament of $1.75 \mathrm{~mm}$ in diameter is introduced into the fused deposition device (FDM: Blackbot Plus) and was melted with the heat of the printer head (with a nozzle pore of $0.40 \mathrm{~mm}$ ) to the temperature of $210{ }^{\circ} \mathrm{C}$, and deposited in the desired locations. The velocity of the nozzle was $30 \mathrm{~mm} / \mathrm{min}$ and the thickness of the layers was $0.3 \mathrm{~mm}$. In order to study the effect of porosity on the scaffold properties, scaffolds were printed at three different densities (containing about 30, 50 and 70\% porosity).

After obtaining a 3D scaffold and removing possible debris, samples were stored for further evaluation.

\subsection{Examination of samples}

\subsubsection{Porosimetery}

The porosity in the samples has been calculated from equation (1).

$$
\text { Equation (1): } \quad \text { \%Porosity }=\left(1-\frac{\rho_{\text {app }}}{\rho}\right) \times 100
$$

Where, $\rho$ is the density of the rigid polymer and $\rho_{\text {app }}$ is the apparent density of the scaffold, which is calculated by dividing the weight by the apparent volume (the total volume considering the porosities). 


\subsubsection{Microstructure study}

Scanning electron microscopy (SEM: Philips-XL30) was used to investigate the morphology and size of scaffold pores. Given that the polymer is not electrically conductive, its surface was first coated with gold through sputter coating to make the surface of the scaffolds conductive and, prevent the accumulation of electrical charge at the their surface during scanning electron microscopy. The images were prepared by a secondary electron detector.

\subsubsection{Phases analysis}

$\mathrm{X}$-ray diffraction (XRD: Philips, X'pert-MPD) with a source of $\mathrm{Cu}-\mathrm{K} \alpha$ radiation $(\lambda=1.5405$ $\mathrm{A}^{\circ}$ ) at voltage of $40 \mathrm{kV}$ was used to investigate the initial filament phases and also printed scaffolds. The scan was performed at a range of 20 to 80 degrees at the rate of $2 \theta=1 \% \mathrm{~min}$.

\subsubsection{Assessment of mechanical properties}

In order to study the mechanical properties of the samples, a compression test was performed by a Santam STM-150 compression test device with a strain rate of $0.5 \mathrm{~mm} / \mathrm{min}$ at ambient temperature. Compression test samples were prepared with dimensions of $10 \times 10 \times 10 \mathrm{~mm}$ and dimensional tolerance of $\pm 0.5(\mathrm{~mm})$ and, were evaluated mechanically three times and the average values were presented.

\section{Results and Discussion}

The results of porosimetery of the scaffolds are given in Table 1, given that the density of the poly lactic acid polymer of $1.25 \mathrm{~g} / \mathrm{cm}^{3}$.

Table 1: Weight and porosity of printed scaffolds

\begin{tabular}{|c|c|c|c|}
\hline Sample & Weight (gram) & Apparent volume $\left(\mathrm{cm}^{3}\right.$ & Porosity (volume percent) \\
\hline 1 & 0.378 & 1 & 69 \\
\hline 2 & 0.642 & 1 & 49 \\
\hline 3 & 0.892 & 1 & 29 \\
\hline
\end{tabular}

This table shows that the porosity of the samples is 29,49 and 69 volume percent. Porosity values above $50 \%$ are good values for tissue growth in the scaffold and biological fixation. The three-dimensional design and the printed pieces (before cutting into one centimeter samples) 
have been presented in Fig. 1 and, represents the regular distribution of cavities along the scaffold.
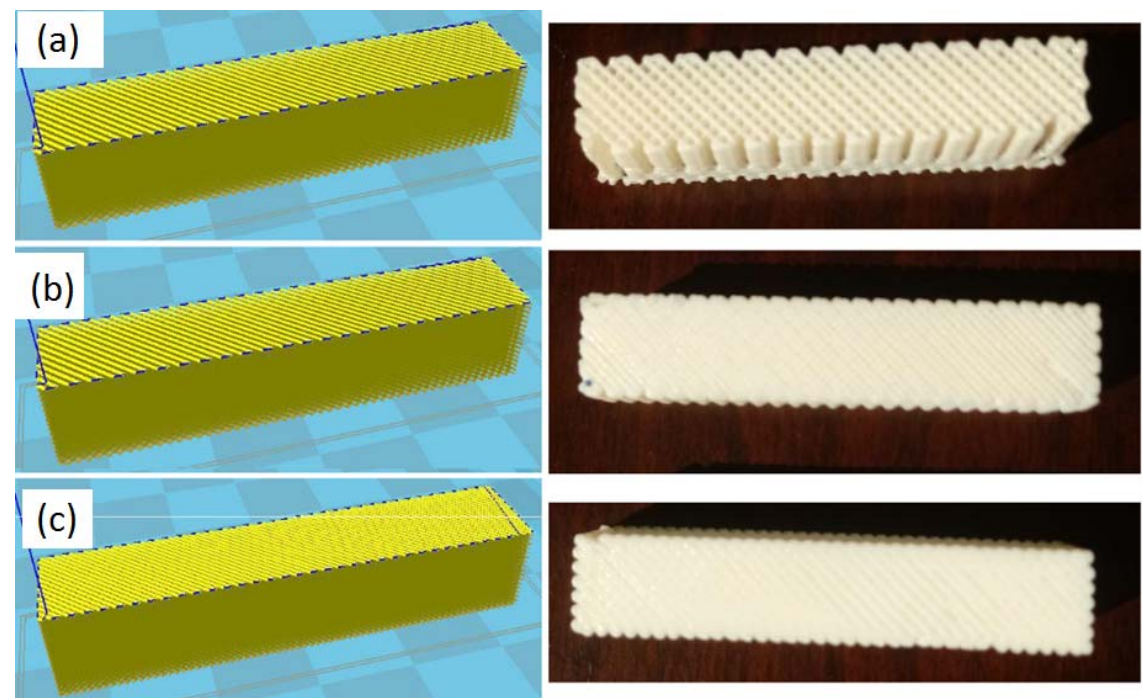

Figure 1: Three-dimensional design and printed poly lactic acid piece with fused deposition modeling with porosity of a) 69 , b) 49 and c) 29 percent

The images of scanning electron microscopy of scaffolds have been presented in Fig. 2. The parallel view of the $\mathrm{Z}$ axis (height during 3D printing) shows that there are regular and completely interconnected cavities in the polymer matrix. Open porosity in biological scaffolds helps to nourish and remove waste materials surrounding the implant and help to accelerate the tissue healing process [8]. Therefore, the fused deposition modeling is an appropriate method for making polymer scaffolds for bio application.
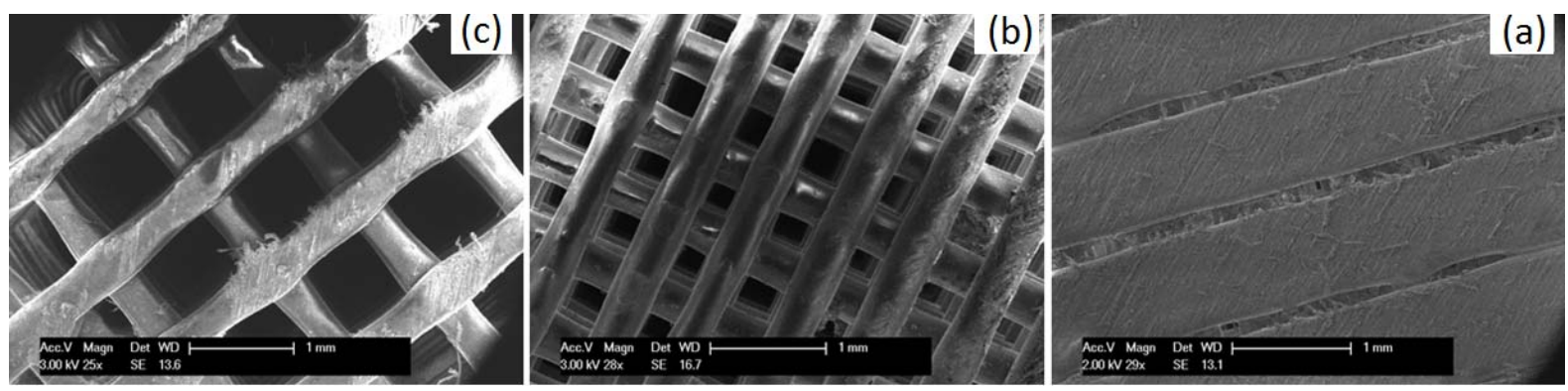

Figure 2: Images of scanning electron microscopy of the poly lactic acid scaffold in porosity values of: a) 29 , b) 49 and c) 69 percent

In this figure, it is observed that the morphology of the cavities is square and regular and their size increases with decreasing porosity. As the size of the cavities of the scaffolds 
containing 29,49 and $69 \%$ of porosity is about 80,250 and 600 micrometers. It is noted that scaffold cavities containing 49 and $69 \%$ porosity are interconnected and suitable for bone tissue engineering.

Since the heating can change the phase or the crystallinity of the polymers, therefore, the phases of the initial poly lactic acid filament (not printed) and printed porous scaffold were studied using X-ray diffraction (X-ray diffraction) test and, the results have been presented in Fig. 3. The results show that the X-ray diffraction pattern did not change before and after the printing process and, this process does not change the properties of the polymer. Also, both patterns, due to wide and extended peak in the range of 17 degrees, indicate the amorphous structure of the polymer which is the feature of polys lactic acid polymer of DL-type (DLPLA), this type of poly lactic acid has less resistance to hydrolysis than L-PLA [9]. The polymer amorphous structure has not been crystallized due to the printing process.

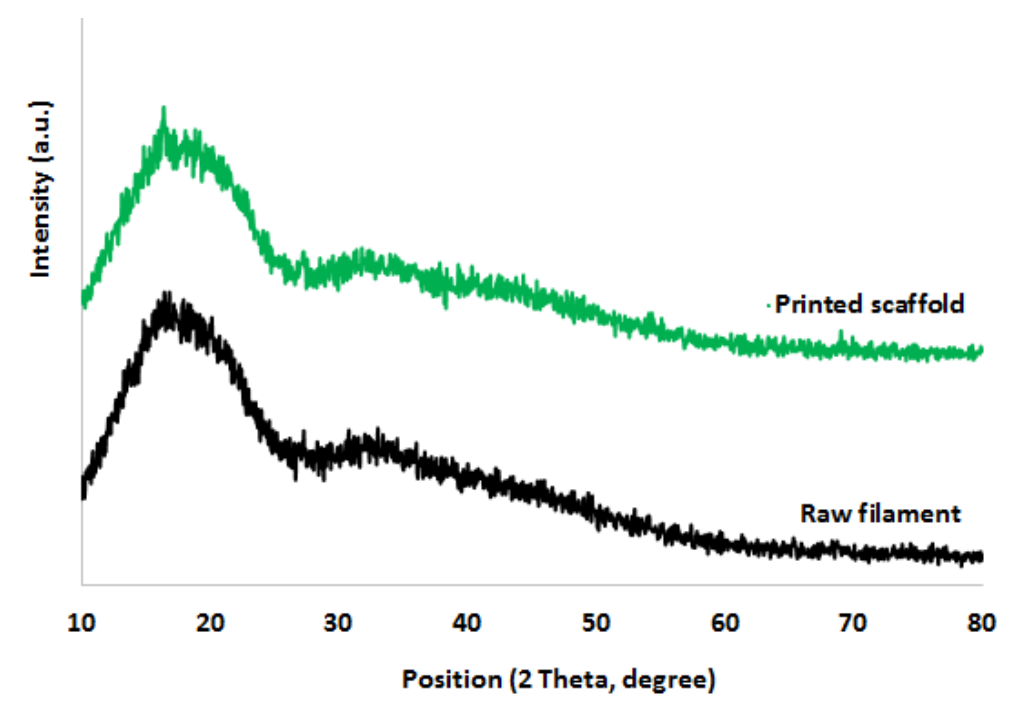

Figure 3: X-ray diffraction patterns of: a) primary filament, b) printed scaffold

The results of the compression test presented in Fig. 4 show that with increasing porosity from 29 to 49 and 69 percent, the plateau stress of scaffold decreased from $41.1 \pm 0.2$ to 29.3 \pm 0.1 and $9.2 \pm 0.1 \mathrm{MPa}$, respectively. The quantitative values of these graphs were calculated according to the ISO 13314 standard. Stress-strain diagram of porous scaffolds consists of three components:

a) Elastic linear region: The region in which the scaffold exhibits non-permanent deformation. 
b) Plateau stress: The permanent deformation range in which the cavities collapsed layer by layer and accumulated.

c) Densification region: When all cavities are compressed, subsequent strains causes a sudden increase in stress.

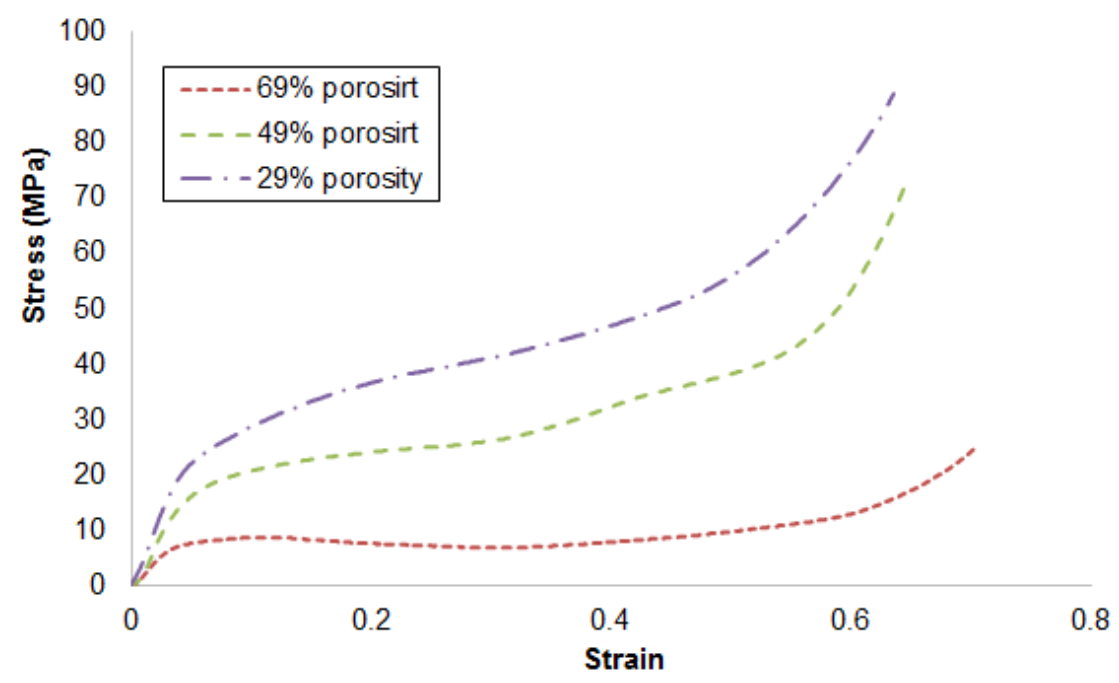

Figure 4: Stress-strain diagram of poly lactic acid scaffolds containing different porosities

The elastic coefficients of the biodegradable poly lactic acid polymer containing 29, 49 and $69 \%$ of porosity are $502.7,537.7$ and $483.3 \mathrm{MPa}$, respectively. It can be seen that with the porosity of the sample, these values are reduced to $69 \%$ of rigid values and have got close to the bone tissue values (about 3-20 MPa [11] and 3000 [12] MPa, respectively).

Therefore, the elastic modulus and strength of porous polymer scaffolds can easily be adjusted proportional to the surrounding tissue and it can be used as a scaffold to repair bone tissue. Research results of other researchers, such as Mohseni et al. [13], also indicate the ability of a 3D polymer scaffold to cell growth and differentiation. Of course, in addition to mechanical and chemical properties, the degradation rate of the three-dimensional scaffold must also be adjusted according to the adjacent rebuild rate $[14,15]$ that, the degradation rate will be further investigated.

\section{Conclusion:}


In this research, three-dimensional porous poly-lactic acid (PLA) scaffolds were prepared by fused deposition modeling (FDM) with 29,49 and $69 \%$ of porosity. The results of the evaluation of mechanical properties show that the mechanical properties of the scaffolds produced were in the range of bone strength, which is reduced by increasing porosity from about 41 to $9 \mathrm{MPa}$, and its mechanical properties are easily adjustable with adjacent stiff tissue. The microstructure study also shows that the size of regular square cavities decreases with decreasing porosity and the porosity should be proportional to the penetration ability of the bone cell and tissue.

\section{Conflicts of interest}

There is no conflict of interest.

\section{References}

1- Khodaei Mohammad, Meratian Mahmood, Savabi Omid, Fathi MohammadHossein. The effect of sintering temperature on the properties of porous titanium implant manufactured by space holder method, Journal Advanced Processes in Materials Engineering, 2015, 9(3):1-9.

2- Hosseini Shahin, Sadrnezhaad Sayed Khatiboleslam, Ekrami Aliakbar, Phase transformation behavior of porous NiTi alloy fabricated by powder metallurgical method, Materials Science and Engineering C, 2009, 29(7): 2203-2207.

3- Corcione Carola Esposito, Gervaso Francesca, Scalera Francesca, Padmanabhan Sanosh Kunjalukkal, Madaghiele Marta, Montagna Francesco, Sannino Alessandro, Licciulli Antonio, Maffezzoli Alfonso, Highly loaded hydroxyapatite microsphere/ PLA porous scaffolds obtained by fused deposition modelling, Ceramics International, https://doi.org/10.1016/j.ceramint.2018.07.297

4- Lin Weiyi, Shen Hongyao, Xu Guanhua, Zhang Linchu, Fu Jianzhong, Deng Xiaolei, Single-layer temperature-adjusting transition method to improve the bond strength of 3D-printed PCL/PLA parts, Composites Part A, 2018, 115: 22-30.

5- Kun Krisztián, Reconstruction and development of a 3D printer using FDM technology, Procedia Engineering, 2016, 149: 203-211 
6- Rinaldi Marianna, Ghidini Tommaso, Cecchini Federico, Brandao Ana, Nanni Francesca, Additive layer manufacturing of poly (ether ether ketone) via FDM, Composites Part B (2018), doi: 10.1016/j.compositesb.2018.03.029.

7- Gomez-Gras Giovanni, Jerez-Mesa Ramón, Travieso-Rodriguez J. Antonio, LlumaFuentes Jordi, Fatigue performance of fused filament fabrication PLA specimens. Materials \& Design (2017), doi:10.1016/j.matdes.2017.11.072

8- Wang Hao, Zhi Wei, Lu Xiong, Li Xiaohong, Duan Ke, Duan Rongquan, Mu Yandong, Weng Jie, Comparative studies on ectopic bone formation in porous HA scaffolds with complementary pore structures, Acta Biomaterialia (2013), doi: http://dx.doi.org/10.1016/j.actbio.2013.05.026

9- Tan Lili, Yu Xiaoming, Wan Peng, Yang Ke, Biodegradable Materials for Bone Repairs: A Review, J. Mater. Sci. Technol., 2013, 29(6): 503-513.

10- Bhatia Sujata K, Biomaterials for Clinical Applications, Springer New York Dordrecht Heidelberg London, doi: 10.1007/978-1-4419-6920-0.

11- Ostrum, Robert F, Biomechanics in Orthopedic Trauma. Bone Fracture and Fixation, The Journal of Bone and Joint Surgery, 1994, 76(9): 1438-1438.

12- Wieding Jan, Wolf Andreas, Bader Rainer, Numerical Optimization of Open-Porous Bone Scaffold Structures to Match the Elastic Properties of Human Cortical Bone, Journal of Mechanical Behavior of Biomedical Material, 2014, 37: 56-68.

13- Mohseni Kouchesfahani Homa, Sanamiri Khadije, Hashemitabar Mahmoud, Application of Alginate capsules as a three dimensional scaffold for differentiation of Wharton's Jelly Mesenchymal stem Cells to definitive endoderm, Arak Medical University Journal, 2014, 17(85): 54-66.

14- Wan Lu, Zhang Yanhua, Jointly modified mechanical properties and accelerated hydrolytic degradation of PLA by interface reinforcement of PLA-WF, Journal of the Mechanical Behavior of Biomedical Materials, 2018, 88: 223-230.

15- Simmons Heather, Kontopoulou Marianna, Hydrolytic degradation of branched PLA produced by reactive extrusion, Polymer Degradation and Stability, 2018, 158: 228237. 